\title{
Transverse Effects under Four-Wave Coupling in the Nonlinear Fabry-Perot Interferometer
}

\author{
O. G. Romanov and A. L. Tolstik \\ Belarussian State University, Minsk, 220050 Belarus \\ Received July 3, 2001
}

\begin{abstract}
Specific features of the light-beam transverse structure transformation in the process of four-wave coupling in the Fabry-Perot interferometer with a resonant nonlinearity under conditions of bifurcation of the symmetry breaking and optical bistability are analyzed. The asymmetric regimes of the interaction, characterized by a difference both in the integrated transmission functions of the nonlinear interferometer for two light beams with equal intensities and in the spatial intensity distribution at the exit of the interferometer, are considered. (C) 2002 MAIK “Nauka/Interperiodica”.
\end{abstract}

The nonlinear Fabry-Perot interferometer (FPI) is a typical example of an optical system which shows diverse effects of spatio-temporal self-organization resulting from interaction between the light beams in combination with different types of feedback (external feedback due to reflection from resonator's mirrors and distributed transverse feedback related to light diffraction) $[1,2]$. The intracavity parametric interaction between the coherent light beams provides an additional type of distributed feedback upon scattering of the waves by dynamic gratings recorded in the volume of the medium. In particular, when the two light beams are incident on the input mirror of the nonlinear FPI at small angles, symmetrically with respect to the normal, the four-wave coupling (FWC) is realized inside the resonator. Under these conditions, new types of optical bistability may show up: $S$ - and $N$-, and "butterfly"-type bistability [3], as well as asymmetric bistability [4].

In this paper, on the basis of the methods of theoretical and numerical simulation, we study the formation of the transverse spatial structures of the light fields under conditions of intracavity FWC. The treatment is based on the mean-field theory for a high-Q resonator with a two-level resonance medium and includes the effects of the transverse and longitudinal distributed feedback between the light beams in the volume of the resonator. We analyze specific features of the light beam transformation under conditions of violation of the symmetry of the interferometer transmission with allowance for the diffraction mechanism of the transverse interaction. We will also determine the regularities in formation of asymmetric (coupled and localized) transverse structures of the light field at the exit of the interferometer.

The dynamics of the intracavity FWC will be analyzed using, as an example, the resonance nonlinearity of the intracavity layer, within the mean-field approximation for a nonlinear interferometer (small optical thickness of the nonlinear layer $k_{0} L \ll 1$, and low transmittance of the interferometer mirrors $T \ll 1$ ) [5]. Such an approximation is also valid for the problems of parametric interaction in a thin high-Q interferometer. It implies averaging the amplitudes of the interacting fields along the resonator axis [6].

To theoretically analyze the formation of the light field transverse structures under the FWC in the nonlinear interferometer, we will use the system of wave equations for the light field complex amplitudes, obtained in [7] for the two-level model of the resonance medium

$$
\begin{gathered}
t_{R} \frac{\partial E_{1}}{\partial t}=E_{10}-E_{1}+i \Delta_{0} E_{1} \\
+i C \frac{\hat{\alpha}}{\alpha} E_{1}\left(\frac{1+I_{1}}{A_{0}}\right)+i \beta \frac{\partial^{2} E_{1}}{\partial x^{2}}+\alpha_{0}^{\prime} \frac{\partial E_{1}}{\partial x}, \\
t_{R} \frac{\partial E_{2}}{\partial t}=E_{20}-E_{2}+i \Delta_{0} E_{2} \\
+i C \frac{\hat{\alpha}}{\alpha} E_{2}\left(\frac{1+I_{2}}{A_{0}}\right)+i \beta \frac{\partial^{2} E_{2}}{\partial x^{2}}-\alpha_{0}^{\prime} \frac{\partial E_{2}}{\partial x},
\end{gathered}
$$

where $A_{0}=1+2\left(I_{1}+I_{2}\right)+\left(I_{1}^{2}+I_{2}^{2}\right) ; E_{10}$, and $E_{20}$ are the amplitudes of the light waves at the entrance of the interferometer, $E_{1}$ and $E_{2}$ are the amplitudes at the exit of the interferometer, $C=k_{0}(\omega) L / T$ is the cooperative parameter, $k_{0} L$ is the optical thickness of the nonlinear layer, $T$ is the transmittance of the resonator's mirrors, $\Delta_{0}=\left(4 \pi n_{0} L / \lambda-2 m \pi\right) / T$ is the initial detuning of the interferometer from the resonance ( $m$ is an integer), $\beta=$ $\lambda L / 2 \pi T$ is the diffraction parameter, $\alpha_{0}^{\prime}=\alpha_{0} L / 2 \pi T$ is the normalized value of the angle of incidence $\alpha_{0}$ of the light beams, $t_{R}=2 L / v T$ is the transient time of the field in the resonator, $\hat{\alpha}=a+i \alpha=\left(\hat{\Theta}_{12}+\hat{\Theta}_{21}\right) / v p_{21}$ is the 


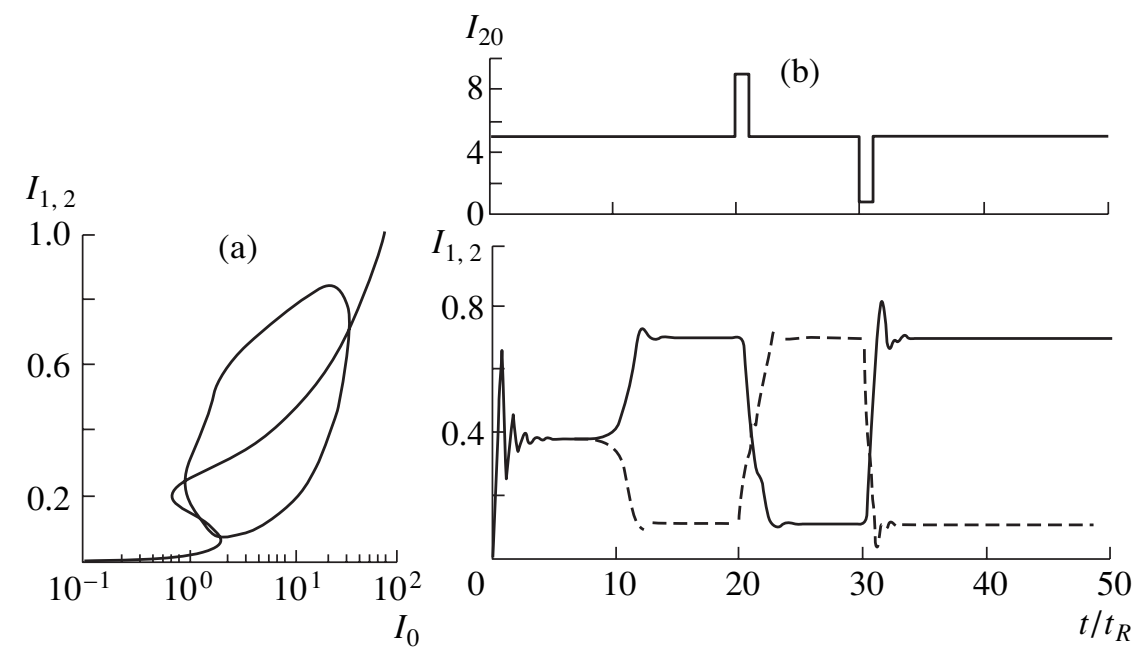

Fig. 1. (a) The steady-state transmission function of the interferometer for two light beams in the region of asymmetric optical bistability, (b) the switching dynamics of the interferometer from the state with symmetric transmission to the state with asymmetric transmission under modulation of the light beam intensity $I_{20}$ at the entrance. The light beam intensities at the entrance are $\alpha I_{0}=5$ $\left(I_{10}=I_{20}=I_{0}\right)$, the cooperative parameter $C\left(\omega_{12}\right)=70$, the initial detuning of the interferometer from the resonance $\Delta_{0}=15$, and detuning of the light beam frequency from the absorption band center is $\xi=-2$.

complex parameter of nonlinearity for the two-level resonance medium [8], $\hat{\Theta}_{i j}(\omega)=\Theta_{i j}(\omega)+i B_{i j}(\omega)$, the coefficients $\Theta_{i j}(\omega)$ are related by the Kramers-Kronig dispersion relations with the Einstein coefficients $B_{i j}(\omega)$ for stimulated transitions, $v$ is the speed of light in the medium, and $p_{21}$ is the total probability of the spontaneous and nonradiative transitions. The light wave intensity is normalized to the coefficient $\alpha=\left(B_{12}+\right.$ $\left.B_{21}\right) / v p_{21}$, which determines the saturation intensity of the resonance medium $\left(I_{\mathrm{sat}}=\alpha^{-1}\right)$.

In the plane-wave approximation, the stationary solution to the system of wave equations (1) can be represented in the form

$$
\begin{gathered}
I_{0}=I_{1,2}\left[\left\{1+\frac{C}{A_{0}}\left(1+I_{1,2}\right)\right\}^{2}\right. \\
\left.+\left\{\Delta_{0}+\frac{a}{\alpha} \frac{C}{A_{0}}\left(1+I_{1,2}\right)\right\}^{2}\right],
\end{gathered}
$$

where the intensities of the input beams are $I_{10}=I_{20}=$ $I_{0}$. Here and in what follows, we consider the model of a two-level resonance medium with coincident Gaussian absorption and emission profiles $\left(\hat{\Theta}_{12} / B_{12}=\hat{\alpha} / \alpha\right.$, $B_{i j}=B_{i j}^{\max } \exp \left(-\xi^{2}\right)$, where $\xi=\left(\omega-\omega_{0}\right) / \delta$ is the detuning, normalized to the line half-width $\delta$ of the light frequency $\omega$ from the absorption band center $\omega_{0}$ of the two-level resonance medium).

Analysis of Eq. (2) allowed us to determine the conditions (the values of the cooperative parameter $C$, frequency detuning $\xi$, and detuning of the interferometer from the resonance $\Delta_{0}$ ), under which the dependence of the output beam intensities $I_{1,2}$ on the intensity of the input beam $I_{0}$ exhibits optical bistability and symmetry breaking in transmission (Fig. 1a). The asymmetric solution is characterized by inequality in the output beam intensities (the annular region in Fig. $1 \mathrm{a}, I_{1} \neq I_{2}$ ). The linear analysis of stability of the interferometer stationary states has shown that the transmission symmetry breaking occurs due to a pitchfork bifurcation of symmetric solution [the $S$-wise dependence of $I_{1,2}\left(I_{0}\right)$ ] [9].

The switching between different transmission states of the interferometer for the two light beams may be implemented by modulating the intensity of one of the beams at the entrance of the interferometer (Fig. 1b). The initial intensity of the light waves was chosen above the intensity of switching of the interferometer into the high-transmission state. As one can see, the transmittance of the interferometer corresponding to the symmetric solution of Eqs. (1) is settled within the time interval $t \sim 5 t_{R}$ after a few oscillations. Then, due to the instability of this solution, the system passes to a state with different transmittances for the two light waves. Note that the rate of development of this process is proportional to the initial difference between intensities of the interacting waves (in the numerical simulation, the relative error in the intensities at the entrance to the interferometer was chosen to be $\sim 10^{-5} \%$ ). After reaching the stable state with a high transmittance for one of the beams $\left(I_{1}\right)$ and low transmittance for the other $\left(I_{2}\right)$, one can change transmission for the light beams by increasing, in a pulsed way, the weaker beam intensity $\left(t=20 t_{R}\right)$ or by decreasing the intensity of the stronger one $\left(t=30 t_{R}\right)$. After oscillating relaxation, the interferometer is switched over to the opposite state. 


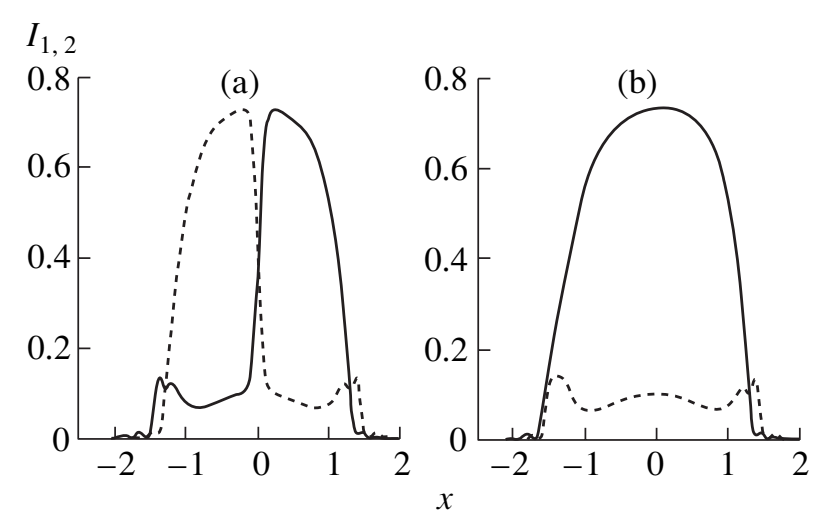

Fig. 2. The steady-state intensity distribution in the transverse profile of the light beams for the symmetric (a) and asymmetric (b) transmission states of the interferometer. The peak intensities of the Gaussian profiles are $\alpha I_{0}=5, \beta=$ $0.01, \alpha_{0}^{\prime}=0.1, \xi=-2$, and $\Delta_{0}=-15$. The transverse coordinate $x$ is normalized to $x_{0}$, which is the half-width of the Gaussian intensity profile at the entrance of the interferometer $I_{10,20}(x, t)=I_{0} \exp \left(-\left(x / x_{0}\right)^{2}\right)$.

The amplitude and duration of the switching signal should be sufficient to transfer the system to the state with almost equal transmittances for the two beams and to reverse the direction of the energy exchange. In this way, the regime of optical switching can be implemented.

To study the effects of transverse distribution, controlled by the light diffraction and angles of incidence of the light beams on the input mirror of the resonator, we have made a numerical simulation of the system of wave equations (1) by constructing the difference-differential approximation based on the two-layer explicit scheme and by using the predictor-corrector technique. The calculations were performed for typical parameters of a thin $(L \sim 1 \mathrm{~mm})$ high-Q $(R \sim 0.01)$ interferometer for the light beam with the wavelength $\lambda \approx 0.63 \mu \mathrm{m}$. The half-width of the beam was $x_{0} \sim 1 \mathrm{~mm}$, with the angles of incidence of the light beams being varied within $0.5^{\circ}-5^{\circ}$.

Consider, first, the defocusing-type nonlinearity, which is realized when the laser beam frequency is detuned to the longer wavelength part of spectrum with respect to the absorption band center. For the symmetric transmission state, the calculated transverse intensity profiles of the light beams are characterized by the mirror symmetry with respect to the beam center at $x=$ 0 and have the same integrated intensity (Fig. 2a). Note that the intensity of each light beam has the highest value in the half-plane from which the beam is incident upon the input mirror of the FPI, i.e., the light beams exhibit a "repulsive" behavior. The energy transfer between different regions of the transverse profiles occurs due to the pitchfork bifurcation and promotes formation of the "barrier" in the central part of the transverse profile $(x=0)$. As was shown by numerical calculations, when the symmetry breaking of the FPI transmission in unstable, the regime of "repulsion" of the light beams is typical of the defocusing-type nonlinearity of the intracavity medium.

To switch the FPI from symmetric to asymmetric transmission, one has, as in the plane-wave approximation, to increase in a pulsed way the intensity of the weaker beam. After the transient process, transverse profiles with substantially different, both local and integrated, intensities arise (Fig. 2b).

Formation of the light field transverse structure strongly depends on the angle of incidence of the light beams upon the interferometer's input mirror. As follows from analysis of the problem of oblique incidence of a light beam on the FPI, as the angle of incidence with respect to the normal of the input mirror increases, the intensity peak shifts along the wave vector and, at a certain angle, intersects the axis $x=0$ [10]. For our case of two-beam interaction, this means that the regimes opposite to the "repulsive" one, demonstrated above, may be realized. The numerical simulation has shown that as the coefficient $\alpha_{0}^{\prime}$, proportional to the angle of incidence, increases, the steady-state intensity profiles change as follows: the switching front between the beams in the vicinity of the axis $x=0$ disappears and a large-scale localized structure arises. In this case, the light beams are characterized, as before, by a spatial distribution with a mirror symmetry with respect to the $x=0$ axis. The crucial factors in formation of the "bound" state of the two light beams are the transverse drift of radiation in the interferometer due to an oblique angle of incidence of the light beams and the energy exchange between the beams due to instability in the symmetry breaking. It is natural to suggest that at a certain angle of incidence, such a structure should transform due to a shift of the peak of each beam to the halfplane opposite to the one in which the incidence occurs. Such a transformation is illustrated in Fig. 3 for $\alpha_{0}^{\prime}=$ 1.27. The transverse structure of the beams is seen to transform from the unstable bound state (Fig. 3a), when the maxima of the two beams in the vicinity of the axis $x=0$ practically coincide, to the state of two independent beams with well separated intensity peaks (Fig. 3b). In the course of transformation of the light beam transverse structure, the energy is transferred virtually completely from the central maxima to peripheral ones. The energy transfer between the peaks separated by two half-widths of a Gaussian profile is rather small, and the stationary state thus realized is stable.

At large angles of incidence of the light beams on the FPI, the effective time of their interaction decreases due to increasing rate of lateral drift for each of the beams. In this case, the light beams pass through each other practically with no energy exchange and without formation of the "bound state." The arising stationary profiles are characterized by a significant distance between their intensity peaks. 

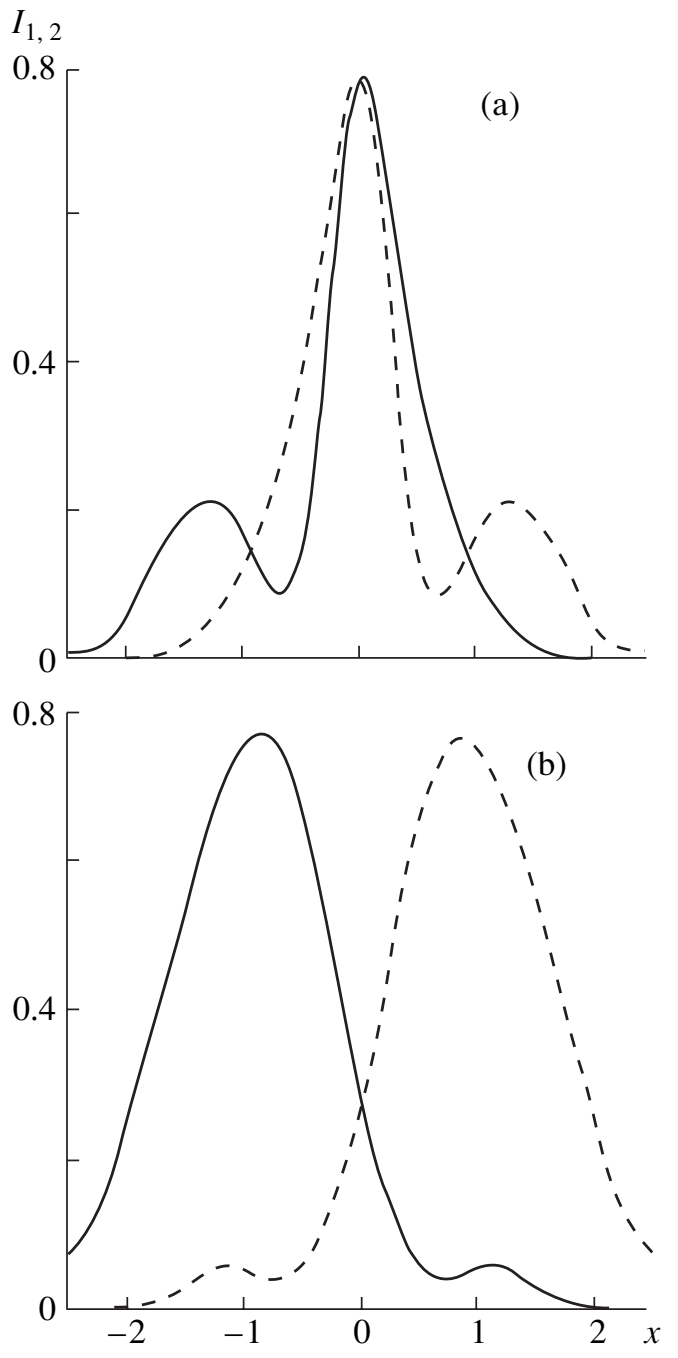

Fig. 3. The intensity distribution in the transverse profile of the light beams at the time moments (a) $t=10 t_{R}$ and (b) $t=$ $100 t_{R}$. The peak intensities of the Gaussian profiles are $\alpha I_{0}=5, \beta=0.01, \alpha_{0}^{\prime}=1.27, \xi=-2$, and $\Delta_{0}=-15$.

An additional factor that complicates the dynamics of the transverse intensity profile of a light beam in the nonlinear interferometer is related to the development of different instabilities that change the field-structure spatial scale. One of the instabilities of this kind is filamentation of the laser beam propagating in a medium with focusing-type nonlinearity, predicted by Bespalov and Talanov [11]. The studies of switching dynamics of the light beam transverse profile in a bistable spatially distributed device have initiated a new trend, specifically, formation of localized structures of the light field. The idea of the diffraction autosoliton in a nonlinear interferometer as a bound state of switching waves was first introduced by Rozanov [2]. The localized transverse structures in nonlinear interferometers are being intensively studied nowadays in view of their possible
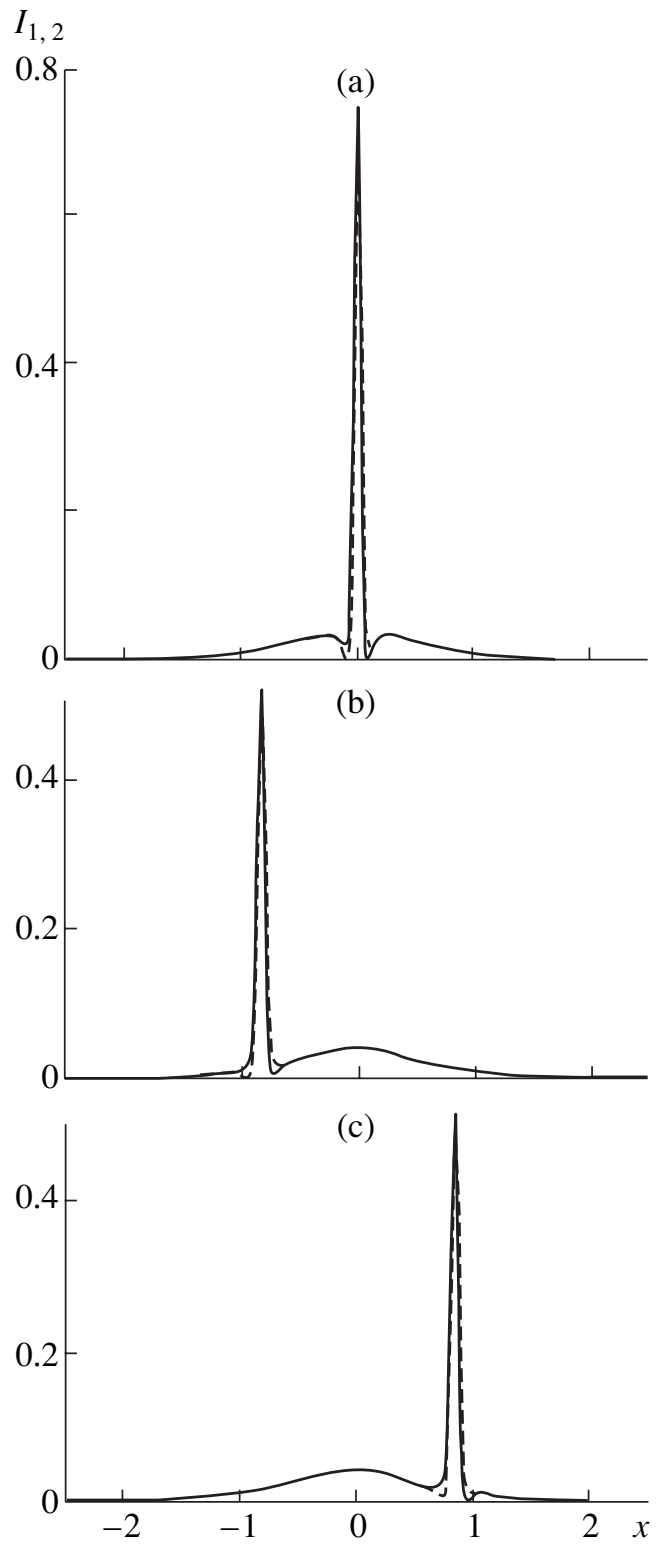

Fig. 4. The steady-state intensity distribution in the transverse profile of the light beams (see the text). The peak intensities of the Gaussian profiles are $\alpha I_{0}=1.75, \beta=0.01$, $\alpha_{0}^{\prime}=0.1, \xi=2$, and $\Delta_{0}=15$.

application in all-optical elements for transforming the laser beams.

As was shown by the results of numerical simulation of the system of Eqs. (1), the soliton-like interaction between the light beams may also show up in the problem of the intracavity FWC. In this case, the input intensity of the light beam, corresponding to the soliton solution, should lie inside the region of optical bistability. If the asymmetric bistability is realized for the same values of the input intensity (the regions of symmetry breaking and optical bistability partially overlap, see Fig. 1), one should expect soliton regimes with a bro- 
ken symmetry of the light beam transverse structure and (or) their integrated intensity. An example of such a self-localized structure is shown in Fig. 4a. As is seen, the bound state of two solitons, mirror-symmetric with respect to the $x=0$ axis, is formed, corresponding to a symmetric solution of system (1). When the controlling optical pulse is applied to one of the beams, the structure of bound asymmetric solitons (Fig. 4a) may be transformed into the states shown in Figs. $4 \mathrm{~b}$ and $4 \mathrm{c}$. In the first case (Fig. 4b), the positive pulse was applied to the first light beam directed to the input mirror of the interferometer from the half-plane $x>0$, and in the second case (Fig. 4c), to the second beam incident from the half-plane $x<0$. As a result, the bound state of the solitons has shifted toward the direction of propagation of the stronger light beam.

Note, in conclusion, that the intracavity FWC demonstrates a number of new effects of self-organization and substantially extends the possibilities of controlling the energy characteristics of light beams and their spatial structure, which may be used in the development of new all-optical elements for the transformation and control of laser beams.

\section{REFERENCES}

1. H. Gibbs, Optical Bistability: Controlling Light with Light (Academic, New York, 1985; Mir, Moscow, 1988).

2. N. N. Rozanov, Optical Bistability and Hysteresis in Distributed Nonlinear Systems (Nauka, Moscow, 1997).

3. S. M. Karpuk, O. G. Romanov, and A. L. Tolstik, Nonlinear Phenomena in Complex Systems 2, 50 (1999).

4. M. Haelterman, P. Mandel, J. Danckaert, et al., Opt. Commun. 74, 238 (1989).

5. O. G. Romanov and A. L. Tolstik, Proc. SPIE 4016, 459 (2000).

6. R. Bonifacio and L. A. Lugiato, Phys. Rev. A 18, 1129 (1978).

7. O. G. Romanov, A. S. Rubanov, and A. L. Tolstik, Litov. Fiz. Zh. 39, 250 (1999).

8. V. V. Kabanov and A. S. Rubanov, Dokl. Akad. Nauk BSSR 24, 34 (1980).

9. I. N. Agishev, E. A. Mel'nikova, O. G. Romanov, and A. L. Tolstik, Vestn. Beloruss. Gos. Univ., Ser. 1 (2001) (in press).

10. M. Haelterman, G. Vitrant, and R. Reinisch, J. Opt. Soc. Am. B 7, 1319 (1990).

11. A. V. Bespalov and V. I. Talanov, Pis'ma Zh. Éksp. Teor. Fiz. 3, 471 (1966) [JETP Lett. 3, 307 (1966)].

Translated by V. Zapasskiǔ 